\title{
Evaluation of Dying Vocal Fold Epithelial Cells by Ultrastructural Features and TUNEL Method
}

\author{
Carolyn K. Novaleski $^{a} \quad$ Masanobu Mizuta ${ }^{b}$ Bernard Rousseau $^{a-c}$ \\ Departments of ${ }^{\mathrm{a}}$ Hearing and Speech Sciences and ${ }^{\mathrm{b}}$ Otolaryngology, Vanderbilt University School of Medicine, \\ and ${ }^{C}$ Department of Mechanical Engineering, Vanderbilt University, Nashville, Tenn., USA
}

\section{Key Words}

Apoptosis · Cell death · Epithelium · Necrosis · Vocal fold

\begin{abstract}
Cell death is a regulated mechanism of eliminating cells to maintain tissue homeostasis. This study described 2 methodological procedures for evaluating cell death in the epithelium of immobilized, approximated and vibrated vocal folds from 12 New Zealand white breeder rabbits. The gold standard technique of transmission electron microscopy evaluated high-quality ultrastructural criteria of cell death and a common immunohistochemical marker, the terminal deoxynucleotidyl transferase dUTP nick end labeling method, to confirm cell death signaling. Results revealed that ultrastructural characteristics of apoptotic cell death, specifically condensed chromatin and apoptotic bodies, were observed after vocal fold vibration and approximation. Although episodes of necrosis were rare, few enlarged cell nuclei were present after vibration and approximation. The vocal fold expresses an immunohistochemical marker for apoptosis along the apical surface of the epithelium. This study provides a solid foundation for future investigations regarding the role of cell death in vocal fold health and disease.

(c) 2016 S. Karger AG, Basel
\end{abstract}

\section{Introduction}

Cell death, a fundamental process during turnover, is characterized by terminating and eliminating cells [Denecker et al., 2001; Pellettieri and Sanchez Alvarado, 2007]. Apoptosis is a common form of programmed cell death that is observed in normal structures and development [Elmore, 2007]. Cysteine-aspartic proteases, or caspases, control apoptosis. The apoptotic cascade is triggered when an initiator caspase is activated, followed by downstream effector caspases (i.e. caspase- 3 and caspase-7). As caspases cleave their substrates, caspase-activated deoxyribonuclease (DNase) causes the hallmark characteristic of DNA fragmentation in cells undergoing

\section{Abbreviations used in this paper}

DAPI 4',6-diamidino-2-phenylindole

DNase deoxyribonuclease

$3^{\prime}$-OH free $3^{\prime}$-hydroxyl ends

PBS phosphate buffered saline

tDt terminal deoxynucleotidyl transferase

TEM transmission electron microscopy

TUNEL terminal deoxynucleotidyl transferase dUTP nick end labeling

\section{KARGER}

E-Mail karger@karger.com

www.karger.com/cto
(C) 2016 S. Karger AG, Basel Carolyn K. Novaleski, PhD

Monell Chemical Senses Center

3500 Market Street

Philadelphia, PA 19104-3308 (USA)

E-Mail cnovaleski@monell.org 
apoptosis [Lalaoui et al., 2015]. Additional evidence of apoptosis includes cell shrinkage, pyknosis, karyorrhexis, cell membrane blebbing and apoptotic body formation and phagocytosis [Majno and Joris, 1995; Elmore, 2007]. Given that apoptosis is a highly controlled process, abnormal cell death can have adverse consequences in a variety of human diseases. In contrast to programmed cell death, necrosis is a disordered manner for cells to die that is characterized by cellular swelling, karyolysis and cell membrane rupture [Majno and Joris, 1995; Denecker et al., 2001].

Epithelial tissues undergo frequent cell death and turnover to promote a strong barrier against various stimuli from the external environment [Hooper, 1956; Pellettieri and Sanchez Alvarado, 2007; Watson et al., 2009]. In the larynx, stratified squamous epithelial cells serve as the outermost portion of the true vocal folds, a pair of multilayered membranous folds of tissue responsible for voice production [Gray, 2000]. The sound of voice is produced when the vocal folds approximate and modulate airflow from the lungs, resulting in repeated vibratory cycles [Van Den Berg, 1958]. Periodic vibration of the 3 layers of lamina propria beneath the epithelium is necessary for normal voice production. Because minor disruptions to the lamina propria can perturb vibration and lead to abnormal voice quality (i.e. dysphonia), the integrity of the epithelial barrier is critical to protect the lamina propria and maintain vocal fold health. Moreover, scientists are increasingly focusing on the structure and function of the vocal fold epithelium.

Lungova et al. [2015] reported that, during embryologic development, apoptosis is responsible for terminating murine vocal fold epithelial cells. In vocal fold disease, apoptotic events were observed in primary tumor and resection biopsies from patients with laryngeal squamous cell carcinoma and epithelial hyperplasia [Hellquist, 1997; Hirvikoski et al., 1999]. In addition to the epithelium, studies revealed that cultured human vocal fold fibroblasts undergo apoptosis in response to a stationary environment and biomechanical stimulation [Gaston et al., 2012; Bartlett et al., 2015]. Collectively, these findings provide support that cell death plays a role during vocal fold development, homeostasis and disease.

In contrast to directly detecting cell death, investigators have more commonly described vocal fold injury and disease by alluding to morphological features consistent with dying cells. For instance, benign lesions of the vocal folds were characterized by desquamating epithelial cells, shrinking nuclei and altered chromatin [Kotby et al., 1988; Dikkers et al., 1993; Martins et al., 2010, 2011]. Re- searchers have also described desquamation in vocal fold epithelial tissues exposed to biomechanical trauma and inhaled pollutants [Gray and Titze, 1988; Marcelino and Oliveira, 2005; Rousseau et al., 2011]. However, these studies fail to confirm that such ultrastructural findings are empirical evidence of vocal fold epithelial cell death. To more consistently describe damage to the vocal folds, it is necessary to implement gold standard approaches to effectively communicate the key biological process of cell death during the life cycle of epithelial cells in the vocal folds.

The objective of this study was to extend the current state of knowledge by establishing methods to evaluate dying vocal fold epithelial cells. In particular, this protocol focused on the gold standard technique of transmission electron microscopy (TEM) for evaluating highquality ultrastructural characteristics of cell death. A common immunohistochemical marker of cell death, terminal deoxynucleotidyl transferase dUTP nick end labeling (TUNEL), was used to confirm these results. A secondary purpose was to investigate cell death in response to vocal fold immobilization, approximation and biomechanical vibration. Immobilized vocal folds were harvested from normal rabbits, approximated vocal folds underwent intermittent adduction and abduction and vibrated vocal folds underwent evoked phonation. Results indicated that, just as in other tissues and as previously reported, the epithelial cells of the vocal fold undergo cell death. In response to approximation and vibration, dying cells demonstrated morphological signs primarily consistent with apoptosis. However, few cells revealed ultrastructural evidence of necrosis after vibration. This study provides 2 standard techniques to more accurately evaluate the process of cell death in the vocal fold epithelium, which may serve as a foundation to better characterize the functional role that cell death plays in the vocal folds.

\section{Materials and Methods}

\section{Animals}

Laryngeal tissue specimens were obtained from 12 adult male New Zealand white breeder rabbits weighing 3-5 kg. Animals were anesthetized via intramuscular injections of ketamine $(35 \mathrm{mg} / \mathrm{kg})$, xylazine $(5 \mathrm{mg} / \mathrm{kg})$ and acepromazine $(0.75 \mathrm{mg} / \mathrm{km})$. To maintain anesthetic effects, ketamine $(17.5 \mathrm{mg} / \mathrm{kg})$ and acepromazine $(0.375$ $\mathrm{mg} / \mathrm{kg}$ ) were subsequently administered as necessary. During the entire experimental procedure, the animals' well-being was ensured by continually monitoring heart rate, oxygen saturation level, body temperature and respiratory rate. The Vanderbilt University Institutional Animal Care and Use Committee approved the procedures. Procedures were performed in accordance with the 


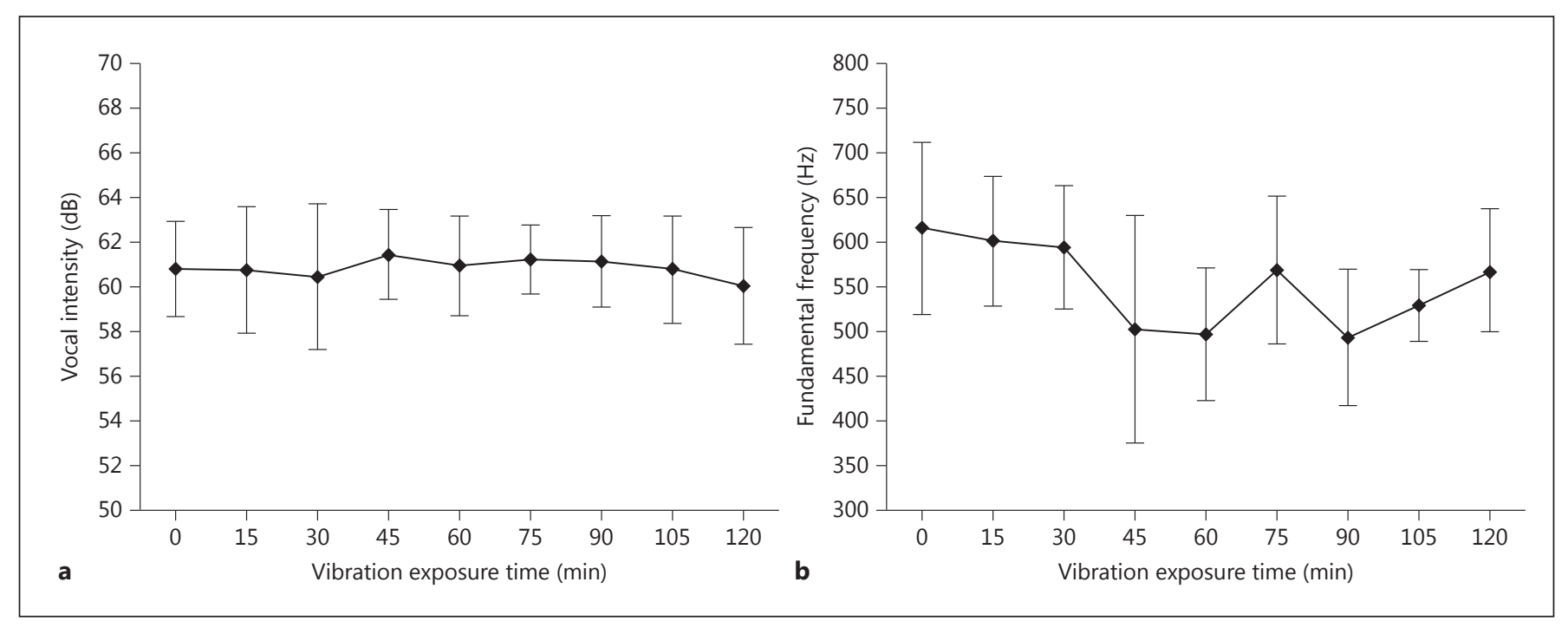

Fig. 1. Stability of acoustic measurements across $120 \mathrm{~min}$ of vibration exposure time (x-axis) for animals that underwent evoked phonation. Data points are mean vocal intensity in $\mathrm{dB}$ sound pressure level (a, $\mathrm{y}$-axis) and mean fundamental frequency in $\mathrm{Hz}$ (b, y-axis). Error bars represent standard deviations of the means.

Public Health Service Policy on Humane Care and Use of Laboratory Animals, National Institutes of Health Guide for the Care and Use of Laboratory Animals and the Animal Welfare Act (7 U.S.C. et seq.).

\section{Procedures}

In vivo Procedures

Cell death in the vocal fold epithelium was evaluated using 3 experimental conditions: immobilization, approximation and vibration. First, normal larynges were harvested to assess cell death in the epithelium of immobilized vocal folds $(n=2)$. Normal tissue was chosen because rabbits do not spontaneously phonate, therefore minimizing the possibility of external vibratory damage. Next, a separate condition included approximated vocal folds at midline with no supplied airflow for $120 \mathrm{~min}(\mathrm{n}=5)$ to examine the effect of laryngeal movement via vocal fold adduction and abduction. To induce this condition, an in vivo surgical procedure was performed as previously described [Ge et al., 2009; Rousseau et al., 2011; Kojima et al., 2014a, 2014b]. In brief, the larynx and trachea were exposed by creating a midline incision from the hyoid bone to the sternal notch. A stable airway was provided via a tracheostomy. Stainless-steel hooked electrodes were inserted into the laryngeal musculature to deliver electrical stimulation. Pulse trains were delivered every $10 \mathrm{~s}$ including $3 \mathrm{~s}$ of electrical stimulation (i.e. adduction) and $7 \mathrm{~s}$ of rest (i.e. abduction).

Finally, a separate condition included vibrated vocal folds (i.e. evoked phonation) via approximation at midline with controlled airflow for $120 \mathrm{~min}(\mathrm{n}=5)$. This vibratory condition was selected based on our laboratory's previous findings which suggest that modal intensity phonation may best represent physiologic vibration. Modal intensity phonation, even up to $120 \mathrm{~min}$, revealed no significant structural disruption to the basement membrane zone and no functional changes in transepithelial resistance [Kojima et al., 2014a]. To evoke phonation, the aforementioned surgical pro- cedures were performed, in addition to inserting a cuffed, inflated endotracheal tube into the trachea approximately $2 \mathrm{~cm}$ below the opening of the glottis. Compressed, humidified airflow was delivered at $37^{\circ} \mathrm{C}$ to the glottis. The combined neuromuscular stimulation and controlled airflow produced sustained, audible phonation of modal intensity. Laryngeal imaging was captured using a rigid endoscope and camera. To detect changes in vocal intensity and fundamental frequency, acoustic signals were recorded at baseline and in 15-min intervals using a Perception 170 Condenser Microphone (AKG, Vienna, Austria) positioned approximately $10 \mathrm{~cm}$ from the opening of the endoscope. Mouth-to-microphone distance and amplitude gain were held constant. Recordings were digitized using the Computerized Speech Lab, model 4500 (KayPENTAX, Montvale, N.J., USA). Acoustic analysis focused on the most representative 1-second central portions of the waveforms. Repeated-measures ANOVA revealed no significant difference in mean vocal intensity across 120 min of vibration $\left(F_{8}=0.60, \mathrm{p}=\right.$ $0.773)$. As displayed in figure $1 \mathrm{a}$, stable intensity values ranged from 60.06 to $61.46 \mathrm{~dB}$ sound pressure level. Similarly, repeatedmeasures ANOVA revealed no significant difference in mean fundamental frequency across 120 min of vibration $\left(F_{8}=1.94, \mathrm{p}=\right.$ $0.087)$. Mean fundamental frequency values ranged from 492 to $615 \mathrm{~Hz}$ (fig. 1b). At the end of all procedures, the animals were sacrificed and their larynges were excised. One vocal fold was used for TEM and the contralateral vocal fold was used for TUNEL method.

Transmission Electron Microscopy

Eleven of the 12 vocal fold specimens were prepared for routine processing for TEM. One vocal fold in the normal condition was not prepared for TEM because this tissue had been used for a separate experiment. For primary fixation, tissue specimens were immersed in $2.5 \%$ glutaraldehyde in $0.1 \mathrm{M}$ sodium cacodylate buffer $(\mathrm{pH} 7.4)$ and then rinsed with $0.1 \mathrm{M}$ sodium cacodylate buffer 
Table 1. Selected ultrastructural categories and justifications for ratings of TEM images

\begin{tabular}{|c|c|c|}
\hline Category & Theme & Justification \\
\hline Cells with small nuclei & cell size & apoptotic cells shrink in size \\
\hline Cells with enlarged nuclei & cell size & necrotic cells swell \\
\hline Cells with rounded shape & cell morphology & shrinking apoptotic cells appear more circular \\
\hline Cells with elongated shape & cell morphology & healthy vocal fold epithelium is stratified squamous \\
\hline Cells with condensed chromatin & cell death & $\begin{array}{l}\text { chromatin in apoptotic nuclei becomes more } \\
\text { concentrated }\end{array}$ \\
\hline Cells with apoptotic bodies & cell death & $\begin{array}{l}\text { apoptotic bodies extrude from cells before undergoing } \\
\text { phagocytosis }\end{array}$ \\
\hline
\end{tabular}

$(5 \min \times 3)$. For secondary fixation, tissue specimens were immersed in $1 \%$ osmium tetroxide in $0.1 \mathrm{M}$ sodium cacodylate buffer ( $1 \mathrm{~h}$ at room temperature) and then rinsed in $0.1 \mathrm{M}$ sodium cacodylate buffer as before. An ethyl alcohol series was used for specimen dehydration. Specimens were immersed in 30, 50, 75, 85 and $95 \%$ ethyl alcohol for 15 min. Final dehydration occurred using $100 \%(\times 3)$ ethyl alcohol for $15 \mathrm{~min}$, and then propylene oxide and $100 \%$ ethanol for $5 \mathrm{~min}$, followed by incubation in $100 \%$ propylene oxide $(\times 2)$ for $15 \mathrm{~min}$ each. Stepwise infiltrations were completed with Epon resin and specimens were embedded in flat molds and allowed to polymerize for $48 \mathrm{~h}$ at $60^{\circ} \mathrm{C}$. Thick sections ( $500 \mathrm{~nm}$ to $1 \mu \mathrm{m}$ ) were initially cut from each block, the region of interest was identified and then ultrathin sections $(70 \mathrm{~nm})$ were cut and placed on 300-mesh copper grids. Grids were postsection stained with $2 \%$ uranyl acetate for 15 min and then with Reynold's lead citrate for $15 \mathrm{~min}$. After TEM preparation, images were captured using a Philips/FEI T12 transmission electron microscope (FEI Company, Hillsboro, Oreg., USA) at low and high magnifications to visualize the apical epithelial cell surface and internal cellular structures.

To initially assess the most common morphological features of cell death, our lead author (C.N.) performed a nonblinded evaluation of TEM images from selected vocal fold tissues that were immobilized $(n=1)$, approximated $(n=2)$ and vibrated $(n=3)$. This preliminary analysis involved subjective and qualitative descriptions of cellular features that are consistent with cell death. These data were used to create 6 categories for a subsequent rating task with TEM images from all 11 specimens. As displayed in table 1 with corresponding justifications, the categories focused on cell size, cell morphology and characteristics specific to cell death. These categories have previously been cited in the literature as evidence of dying cells under visualization by TEM [Elmore, 2007].

Two blinded judges completed a rating task in which they were presented with TEM images. The order of image presentation was randomized. For each specimen, 3 distinct TEM images were included with the following criteria: images were captured at $\times 4,400$ magnification, showed a visible apical surface and contained at least 1 identifiable epithelial cell nucleus. The judges were asked to count the total number of cells in each image and then the number in each of the following 6 categories: (1) small nuclei, (2) enlarged nuclei, (3) rounded cell shape, (4) elongated cell shape, (5) condensed chromatin and (6) apoptotic bodies (table 1). The number of cells in each category was divided by the total number of cells and converted to a percentage. Percentages were averaged across 3 TEM images per specimen for both judges (i.e. a mean of 6 percentage values per specimen). The judges were permitted to refer to the visual examples of each category, increase image magnification, change previous responses and take brief breaks to minimize the effects of fatigue. The first judge (C.N.) trained the second judge (a member of the same lab) in the aforementioned procedures.

To determine if the judges internally rated the TEM images with consistency, the intrarater reliability was evaluated, where the judges repeated the rating task on $50 \%$ of the original TEM images. The order of image presentation was again randomized. For both judges, 2 separate paired-samples $t$ tests and 2 separate Pearson product-moment correlation coefficients were computed. Interrater reliability was evaluated to determine if the judges rated the TEM images similarly to one another. An independent-samples $t$ test and Pearson's product-moment correlation coefficient were computed.

\section{TUNEL Method}

Cryosectioning of all 12 vocal fold tissues specimens was performed. Tissues were immersed in $4 \%$ paraformaldehyde for $24 \mathrm{~h}$, followed by immersion in sucrose for $24 \mathrm{~h}$. Specimens were coated in optimal cutting temperature compound embedding medium (Fisher HealthCare, Houston, Tex., USA), placed in liquid nitrogen, stored at $-80^{\circ} \mathrm{C}$ and sliced into $2-\mu \mathrm{m}$-thick sections; this thickness was selected to ensure that the vocal fold epithelium was preserved during cutting and embedding, as thicker sectioning reduced episodes of damage to the tissue. The TUNEL method was performed in cryosections ( 2 sections/larynx) with the ApopTag ${ }^{\circledR}$ fluorescein in situ apoptosis detection kit [2012] (EMD MILLIPORE International, Inc.). Post-fixed slides were washed with PBS. Slides were pretreated with precooled $30 \mathrm{ml}$ ethanol and $15 \mathrm{ml}$ acetic acid at $-20^{\circ} \mathrm{C}$ for $5 \mathrm{~min}$. Slides were drained and washed with PBS. Equilibration buffer was applied to each laryngeal specimen and incubated at room temperature for $10 \mathrm{~s}$. Workingstrength terminal deoxynucleotidyl transferase ( $\mathrm{tDt}$ ) enzyme was applied to each specimen and incubated in a humidified chamber at $37^{\circ} \mathrm{C}$ for $1 \mathrm{~h}$. Slides were agitated in working-strength stop/wash buffer for $15 \mathrm{~s}$ and incubated at room temperature for $10 \mathrm{~min}$. Slides were washed with PBS and working-strength antidigoxigenin conjugate was added to the specimen surface area. Slides were incubated in a humidified chamber at room temperature for 30 min. Slides were washed and Fluoromount $-G^{\circledR}$ mounting medium containing $4^{\prime}$,6-diamidino-2-phenylindole (DAPI) was applied to the specimens and mounted under a glass coverslip. 


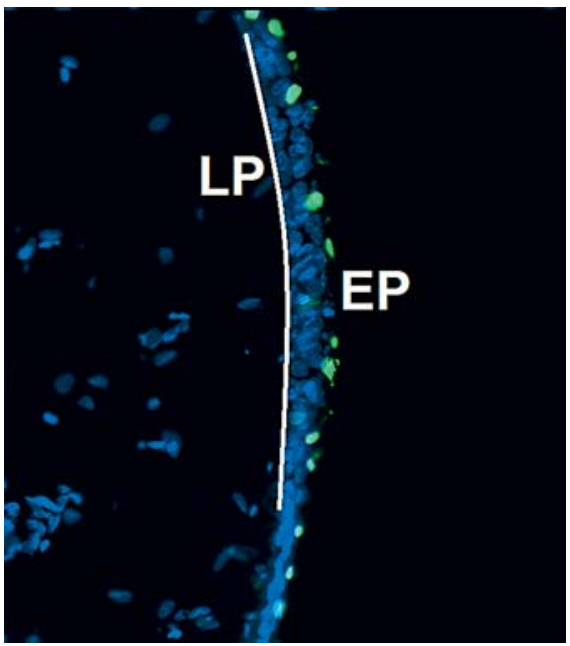

Fig. 2. Image illustrating the region defined as the epithelial layer of the vocal fold during the rating task for TUNEL staining. On the right, the epithelium (EP) faces the apical surface. The white line demarcates the lamina propria (LP) on the left, as determined by a reduction in cells.

Negative and positive controls were created according to the procedures recommended in the ApopTag ${ }^{\circledR}$ fluorescein in situ apoptosis detection kit [2012]. A negative control was performed using a vocal fold tissue specimen exposed to biomechanical vibration for $120 \mathrm{~min}$. The aforementioned TUNEL procedures were performed without active tDt enzyme. For the positive control, a normal vocal fold tissue specimen was pretreated with deoxyribonucleic buffer consisting of $30 \mathrm{~mm}$ Trizma base, $\mathrm{pH}$ 7.2, $4 \mathrm{~mm}$ $\mathrm{MgCl}_{2}$ and $0.1 \mathrm{mM}$ dithiothreitol at room temperature for $5 \mathrm{~min}$, and then $0.2 \mu \mathrm{l}$ DNase was dissolved in $2 \mathrm{ml}$ deoxyribonucleic buffer. DNase solution was applied to the tissue section, incubated at room temperature for $10 \mathrm{~min}$ and rinsed with distilled water. Fluorescent images were captured using a Nikon Eclipse 90i Microscope (NIS-Elements Basic Research, v3.20). Images were acquired of the apical epithelial cell surface of the true vocal fold. Because the images were acquired at varying exposure times, formal quantification procedures were avoided to reduce the risk of error. In lieu of this, a rating task was performed to evaluate TUNEL staining.

The same 2 blinded judges completed a rating task in which they were presented with images of all 12 fluorescent TUNELstained vocal fold specimens at $\times 20$ magnification. The order of image presentation was randomized. The judges selected the degree of TUNEL-positive staining along the entire apical epithelial surface as one of the following classifications per image: no TUNEL-positive cells, a minimal number of TUNEL-positive cells, a moderate number of TUNEL-positive cells and abundant TUNEL-positive cells. TUNEL-positive cells were defined as the amount of visible green staining, as individual cells and/or continuous staining along the epithelium. The epithelium was defined as approximately 2 or 3 layers of blue staining (DAPI) cells that marked a clear outline of the apical vocal fold. Counting discontinued when there was a gap in this region, as indicated by a reduc- tion in cells that likely corresponded to the lamina propria. An example of this boundary distinction is shown in figure 2. The judges were permitted to increase image magnification, change previous responses and take brief breaks to minimize the effects of fatigue. No visual examples of images were provided. Following individual ratings, the first and second judge completed a final consensus rating with 4 images in which there were discrepancies.

To determine if the judges internally rated the fluorescent TUNEL-stained images with consistency, intrarater reliability was evaluated where the judges repeated the rating task on $50 \%$ of the original images. The order of image presentation was again randomized. For both judges, 2 separate paired-samples t tests and 2 separate Pearson product-moment correlation coefficients were computed to investigate the strength of the associations between the original ratings and the repeated ratings. Interrater reliability was evaluated to determine if the judges rated the images similarly to one another. An independent-samples t test and a Pearson product-moment correlation coefficient were computed.

\section{Results}

\section{Intrarater and Interrater Reliability}

Ratings of TEM Images

For the first judge, there was no significant difference between the original ratings versus $50 \%$ of the repeated ratings $\left(t_{101}=0.32, \mathrm{p}=0.752\right)$. Original and repeated ratings were strongly and positively correlated $\left(r_{100}=0.79\right.$, $\mathrm{p}=0.000)$. For the second judge, although there was a significant difference between the original versus repeated ratings $\left(t_{101}=2.52, \mathrm{p}=0.013\right)$, original and repeated ratings were strongly and positively correlated $\left(r_{100}=\right.$ $0.87, \mathrm{p}=0.000)$. For interrater reliability, there was no significant difference between ratings from the first and the second judge $\left(t_{394}=-0.57, \mathrm{p}=0.572\right)$. In addition, ratings from the first judge were positively correlated with ratings from the second judge $\left(r_{196}=0.21, \mathrm{p}=0.003\right)$.

\section{Ratings of Fluorescent TUNEL-Stained Images}

For the first judge, there was no significant difference between the original ratings versus $50 \%$ of the repeated ratings $\left(t_{5}=-1.00, \mathrm{p}=0.363\right)$. Original and repeated ratings were strongly and positively correlated $\left(r_{4}=0.86\right.$, $\mathrm{p}=0.029$ ). For the second judge, there was no significant difference between the original versus repeated ratings $\left(t_{5}=0.00, \mathrm{p}=1.00\right)$. Original and repeated ratings were strongly and positively correlated $\left(r_{4}=0.87, \mathrm{p}=0.024\right)$. For interrater reliability, there was no significant difference between ratings from the first and the second judge $\left(t_{2}=0.351, \mathrm{p}=0.729\right)$. In addition, ratings from the first judge were strongly and positively correlated with ratings from the second judge $\left(r_{1}=0.89, \mathrm{p}=0.000\right)$. 

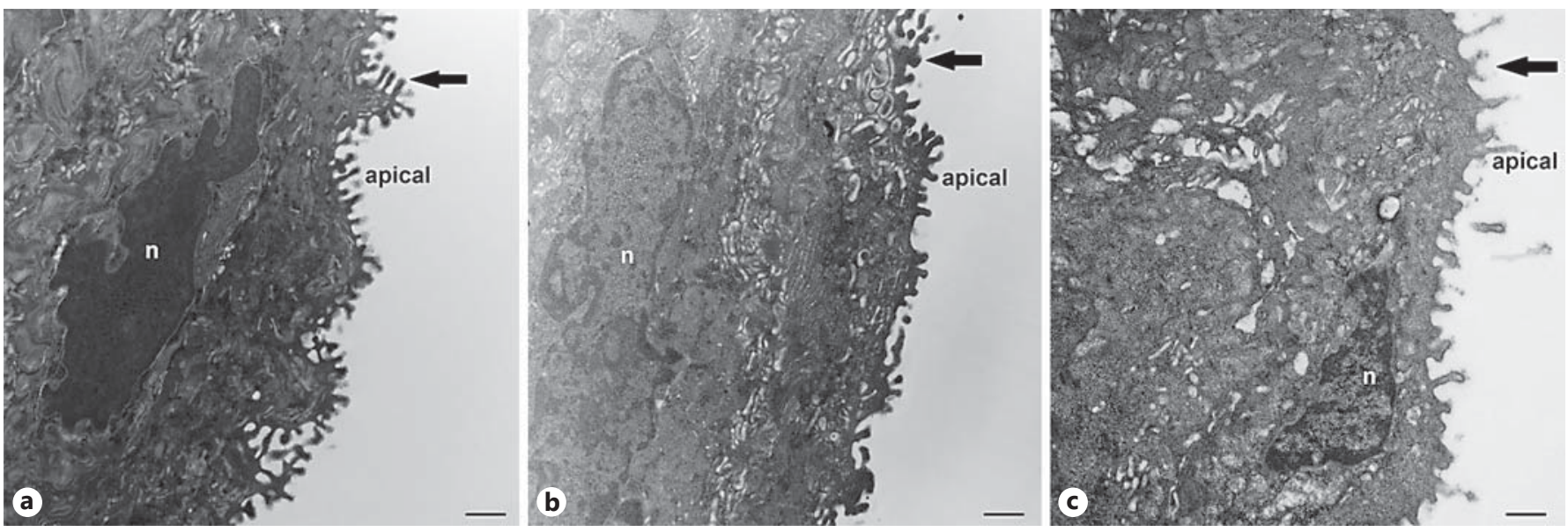

Fig. 3. Analysis of morphology of vocal fold epithelial cell nuclei (n) by TEM. The apical cell surface (on the right) is shown of the true vocal fold epithelium after immobilization (a), approximation for $120 \mathrm{~min}(\mathbf{b})$ and vibration for $120 \mathrm{~min}$ (c). Microvilli (arrows) show a regular shape in immobilized vocal fold tissue, but become increasingly irregular after approximation and vibration. $\times 15,000$. Scale bars $=500 \mu \mathrm{m}$.

Table 2. Summary of ultrastructural characteristics of cell death in immobilized, approximated and vibrated vocal folds

\begin{tabular}{lll}
\hline Immobilized & Approximated & Vibrated \\
\hline Flattened cell shape & flattened cell shape & small nuclei \\
Organized microvilli & less organized microvilli & irregular nuclear envelopes \\
Defined nuclear envelopes & large nuclei & uneven microvilli \\
Large, elongated nuclei & euchromatin & large extracellular space \\
Dark-staining nucleolus & preserved organelles & condensed chromatin \\
Heterochromatin & reduced cell-to-cell contact & preserved organelles \\
Euchromatin & & apoptotic bodies \\
Preserved organelles & & enlarged cells \\
Close cell-to-cell contact & vacuolated cytoplasm \\
& & scarce organelles \\
& & poorly defined membranes \\
\end{tabular}

\section{Ultrastructural Evaluation by TEM}

Preliminary Qualitative Analysis

Table 2 summarizes the major ultrastructural characteristics of cell death across immobilized, approximated and vibrated vocal folds. Normal immobilized vocal fold epithelium comprised approximately 3 layers of cells that demonstrated a flattened morphology consistent with stratified squamous cell shape. The apical membranes of polarized epithelial cells were distinctly outlined with microvilli protruding along the entire length of the surface facing the lumen. Cells showed well-defined nuclear envelopes and large, elongated nuclei. Cell nuclei contained a dark-staining nucleolus and chromatin in the form of tightly coiled heterochromatin and loosely coiled euchromatin (fig. 3a). The cytoplasm and organelles appeared structurally preserved. Adjacent cells were packed closely together, making it challenging to demarcate precise boundaries between cells along the basolateral membranes at lower-magnification fields of view.

Similarly, approximated vocal fold epithelial cells demonstrated a flattened shape. Microvilli of less uniform length covered the apical cell membranes. Large euchromatic nuclei were present (fig. 3b). Although preserved cytoplasm and organelles were appreciated, there 
Fig. 4. Evaluation of ultrastructural features of different stages of apoptotic cell death in vocal fold epithelial cells after biomechanical vibration for $120 \mathrm{~min}$. a Vital cells (asterisks) are located basolaterally with close boundaries between one another, and an apical apoptotic cell (arrow) is shown with a large extracellular space between its neighboring vital cells. Examination of cell nuclei $(n)$ reveals that the apoptotic cell is beginning to shrink in size compared to the vital cells. In the apoptotic cell nuclei, chromatin is condensed and peripheralized. $\times 6,500$. b Several mitochondria $(\mathrm{m})$ are surrounding a pyknotic nucleus (n) of a cell undergoing apoptosis. $\times 11,000$. c An apoptotic cell nucleus (n) begins to break down into several apoptotic bodies (arrows). $\times 11,000$. d A more advanced formation of apoptotic bodies (asterisks) is visible inside the cell cytoplasm (c) and intact plasma membrane of an apoptotic cell (arrow). At this phase, there is no evidence that apoptotic bodies are engulfed by a macrophage, as microvilli continue to protrude from the apoptotic epithelial cell membrane, the shape of which aligns with its neighboring vital cells with visible nuclei (n). $\times 6,500$. Scale bars $=2 \mu \mathrm{m}$.
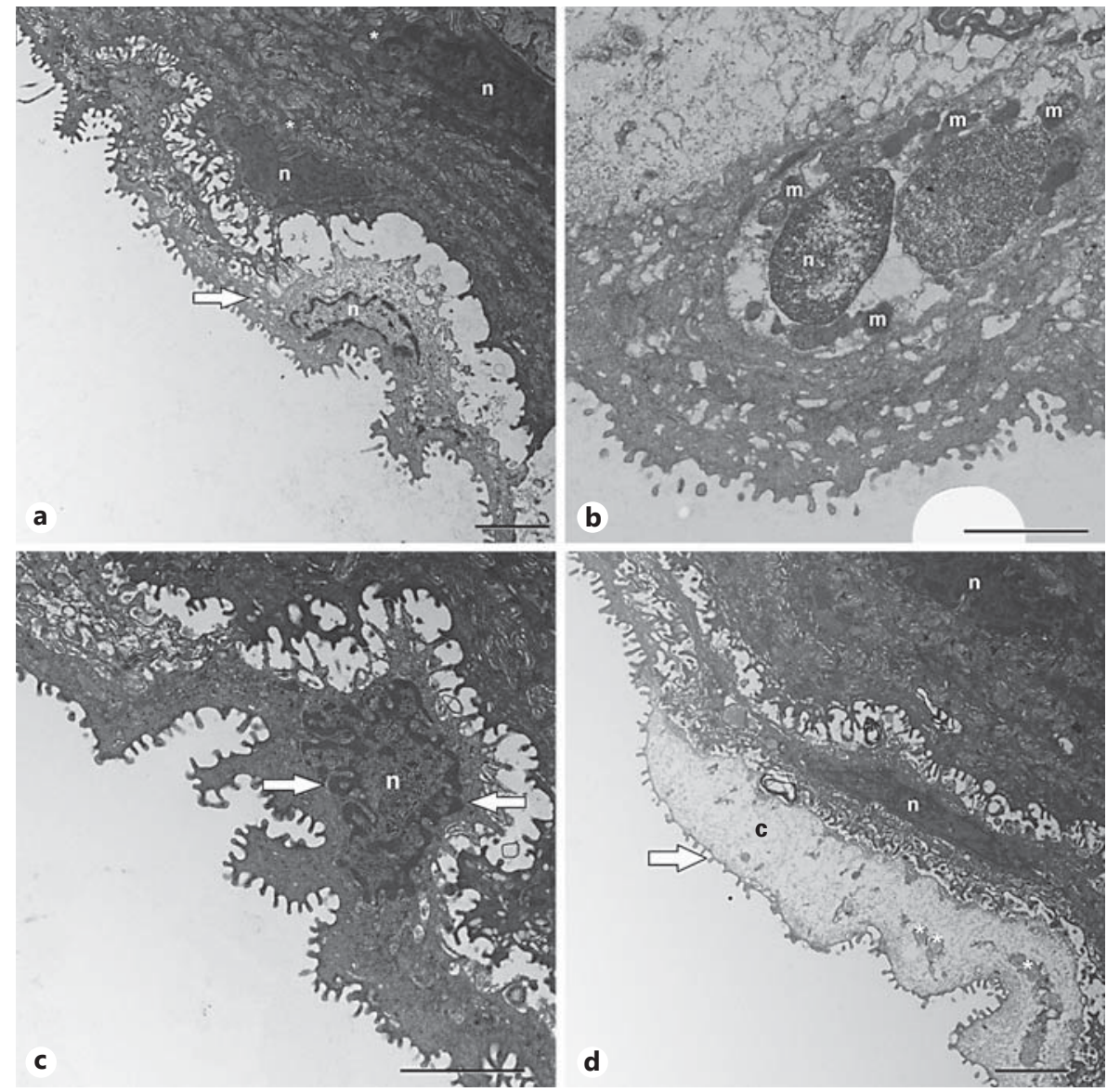

was less cell-to-cell contact between the basolateral domains of the most superficial cells and the apical domains of the next layer of cells beneath. This loose connection appeared to be the result of increased expansion of the tight intercellular junctional complex. However, cell membranes and intercellular contents were maintained.

Finally, vibrated vocal fold epithelial cells were characterized by small nuclei and irregularly shaped, dark-staining nuclear envelopes (fig. 3c). Uneven microvilli projected from the apical cell membranes. Large extracellular spaces separated adjacent cells. Cells showed a circular morphology and appeared to be shrinking inward. Cell membranes were relatively outlined, indicating that the membranes remained intact.

TEM images in figure 4 illustrate the most common morphological features of apoptotic cell death in vocal fold epithelial cells. As shown in figure 4a, apical epithelial cells had condensed, peripheralized chromatin. Organelles were still preserved, particularly mitochondria (fig. 4b). In some instances, cell nuclei began to break down into apoptotic bodies (fig. 4c, d). There was no evidence of engulfment of apoptotic bodies by neighboring cells or macrophages. In addition, several epithelial cells from one vibrated vocal fold showed features consistent with necrotic cell death (fig. 5). Necrosis was characterized by enlarged cells and nuclei compared to the basal epithelial cells located near the basement membrane zone. The necrotic cells had vacuolated cytoplasm, scarce organelles and a poorly defined cell membrane, suggestive of initial membrane rupture (fig. 5a). However, there were no apparent observations of dispersal of cytoplasm into the extracellular space. Clusters of fragmented chromatin were also evident (fig. 5b).

\section{TEM Rating Task}

The rating task of TEM images revealed that the mean percentage of vocal fold epithelial cells with small nuclei was similar across all 3 experimental conditions. As shown in figure $6 \mathrm{a}$, the epithelial cells from animals with 

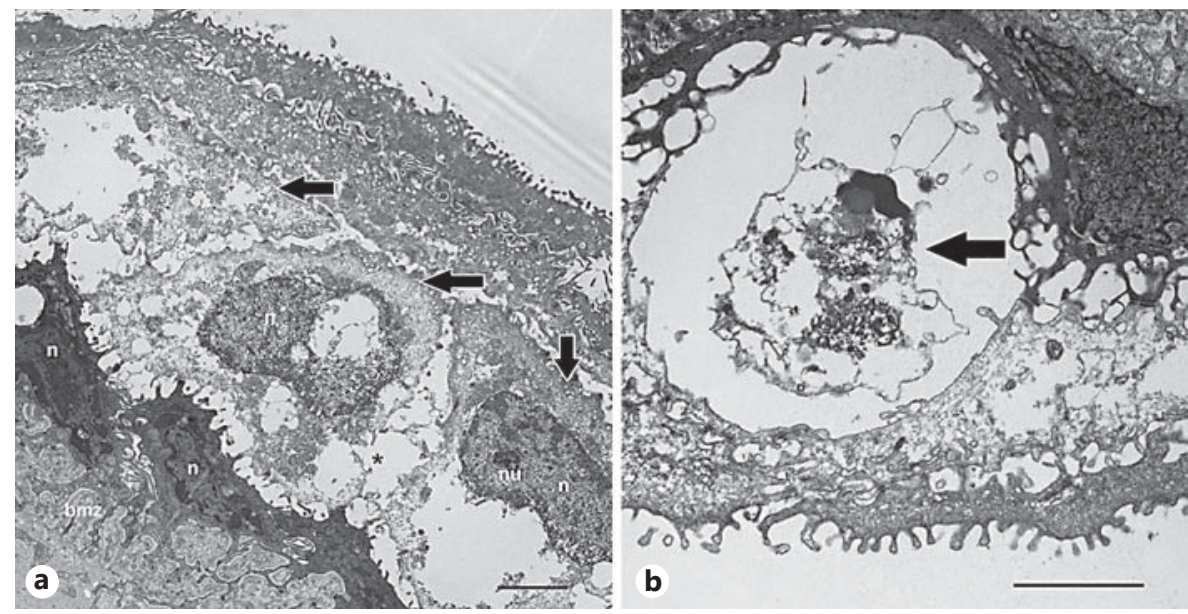

Fig. 5. Evaluation of ultrastructural features of different stages of necrotic cell death in vocal fold epithelial cells after biomechanical vibration for $120 \mathrm{~min}$. a Necrotic cells (arrows) are visibly enlarged in comparison to vital basal epithelial cells located near the basement membrane zone $(\mathrm{bmz})$. Necrotic cells are characterized by vacuolated cytoplasm and few organelles, while the swollen nuclei (n) and nucleolus (nu) remain identifiable. Large extracellular spaces surround the necrotic cells in contrast to vital basal cells which demonstrate closer boundaries to neighboring cells. There is evidence of an emerging ruptured cell membrane (asterisk). $\times 6,500$. $\mathbf{b}$ High magnification microscopy image of an apical necrotic cell showing clusters of chromatin fragmentation (arrow). Edges of the cell membrane are less defined and might indicate the future vulnerability to increased cell membrane permeability. $\times 11,000$. Scale bars $=2 \mu \mathrm{m}$.

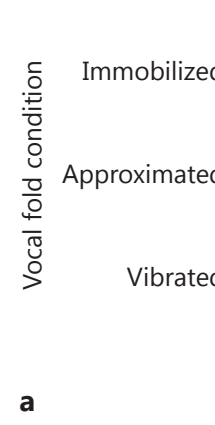

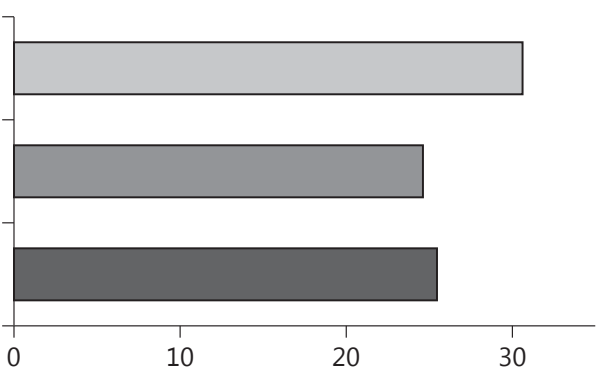

Cells with small nuclei (\%)

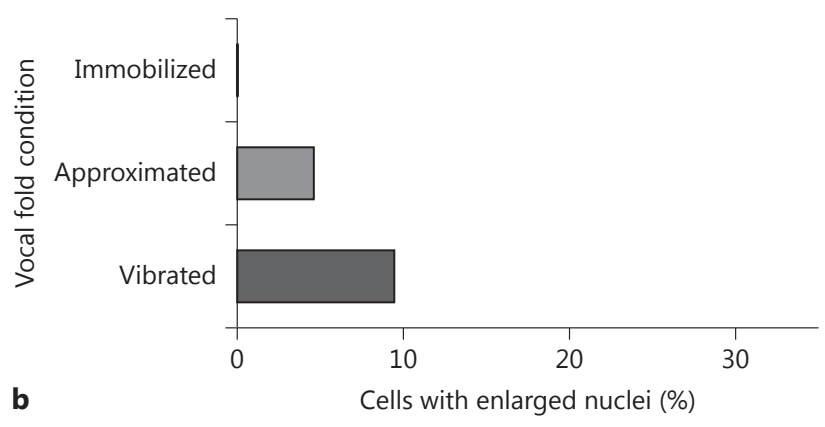

Fig. 6. Mean percentage of vocal fold epithelial cells (x-axis) rated by cell size. Ratings are shown for immobilized (light grey bars), approximated (dark gray bars) and vibrated vocal folds (black bars) for small (a) and enlarged (b) nuclei.

immobilized vocal folds were rated as having the greatest percentage of small nuclei $(30.60 \%)$, followed closely by the vibrated $(25.45 \%)$ and approximated vocal folds $(24.60 \%)$. In contrast, enlarged cell nuclei were ultrastructural features that were rarely observed. Ratings of enlarged nuclei were indicated in only the vibrated (9.44\%) and approximated $(4.60 \%)$ vocal folds. No epithelial cells in the TEM images of immobilized vocal folds were observed to have enlarged nuclei (0\%; fig. $6 \mathrm{~b})$.
Analysis of cell morphology showed that a rounded cell shape was rated highest in the cells of vibrated vocal folds (49.09\%), with lower mean percentages for the approximated $(38.16 \%)$ and immobilized vocal folds (27.82\%; fig. 7a). As expected, there was an inverse trend for the category of elongated cell shape. Specifically, fig. $7 \mathrm{~b}$ shows that an elongated cell shape was rated highest for the immobilized vocal folds (59.96\%) and continued to decrease for the approximated (49.41\%) and vibrated vocal folds (41.05\%). 


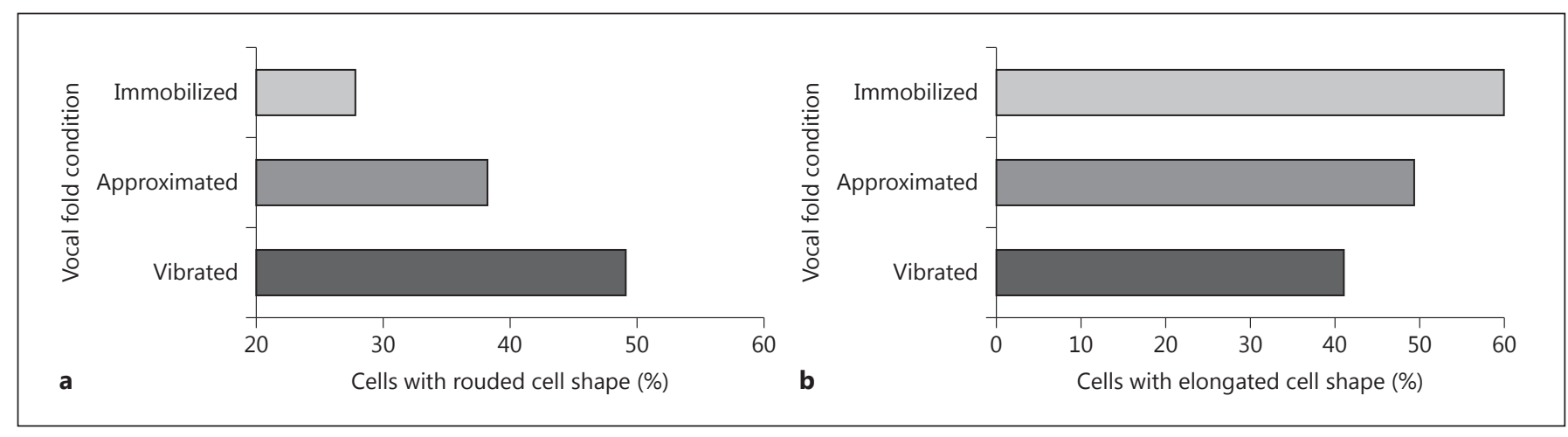

Fig. 7. Mean percentage of vocal fold epithelial cells (x-axis) rated by cell morphology. Ratings are shown for immobilized (light grey bars), approximated (dark gray bars) and vibrated vocal folds (black bars) for a rounded (a) and elongated (b) cell shape.

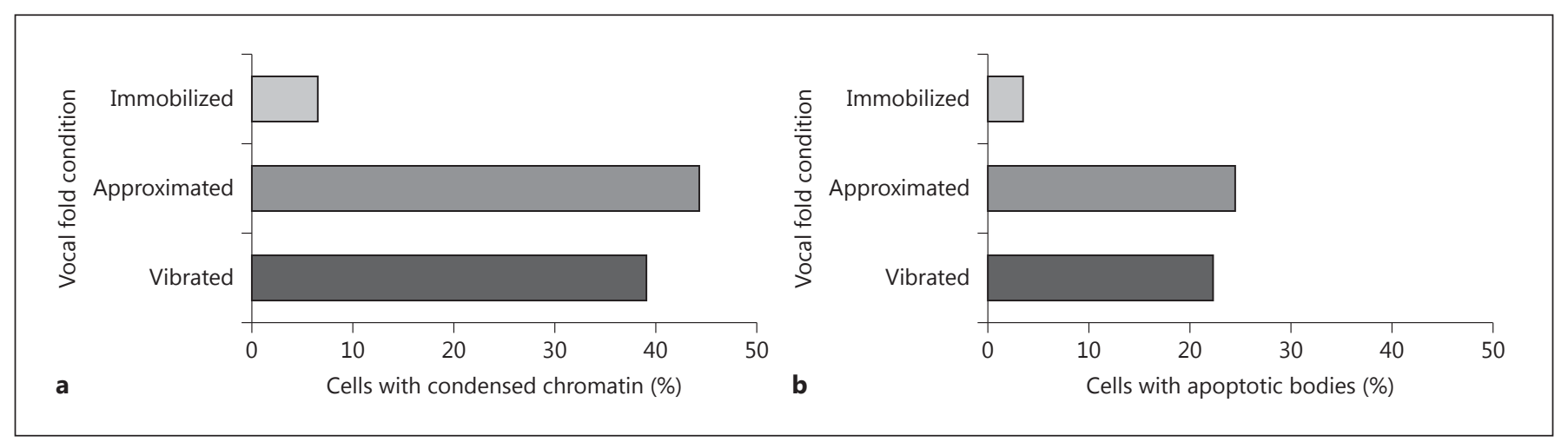

Fig. 8. Mean percentage of vocal fold epithelial cells (x-axis) rated by common features of cell death. Ratings are shown for immobilized (light grey bars), approximated (dark gray bars) and vibrated vocal folds (black bars) for condensed chromatin (a) and apoptotic bodies (b).

Categories related to cell death indicated that approximated and vibrated vocal folds had higher ratings of condensed chromatin (fig. 8a) and apoptotic bodies (fig. 8b) compared to immobilized vocal folds. In particular, the mean percentage of epithelial cells with condensed chromatin was rated highest for the approximated (44.30\%) and vibrated vocal folds (39.14\%), while very few epithelial cells in the immobilized condition showed signs of condensed chromatin (6.61\%). Likewise, the mean percentage of cells with apoptotic bodies was rated highest for the approximated $(24.50 \%)$ and vibrated vocal folds (22.25\%), in comparison to only $3.52 \%$ of epithelial cells from animals with immobilized vocal folds.

\section{Immunohistochemical Detection by TUNEL}

TUNEL revealed the expression of cell death along the apical region of the true vocal fold epithelium. Figure 9

Dying Vocal Fold Epithelial Cells shows representative fluorescent TUNEL-stained vocal fold tissue sections after immobilization (fig. 9a), approximation (fig. 9b), and vibration (fig. 9c) acquired at $\times 20$ magnification. Table 3 displays the mean percentages of the degree of TUNEL-positive cells rated across the 3 experimental conditions. In the immobilized condition $(n=2)$, $50 \%$ of the fluorescent images were rated as displaying no TUNEL-positive epithelial cells and the other $50 \%$ as displaying a moderate number of TUNEL-positive cells. The majority of fluorescent images of the approximated vocal folds were rated as no/a minimal number of TUNEL-positive cells (rated as $40 \%$ of each). The majority of fluorescent images of the vibrated vocal folds were rated as moderate/ abundant TUNEL-positive cells (rated as $40 \%$ of each).

The negative control confirmed no presence of TUNEL staining (fig. 10a). To determine if TUNEL worked properly, a positive control was used to induce 

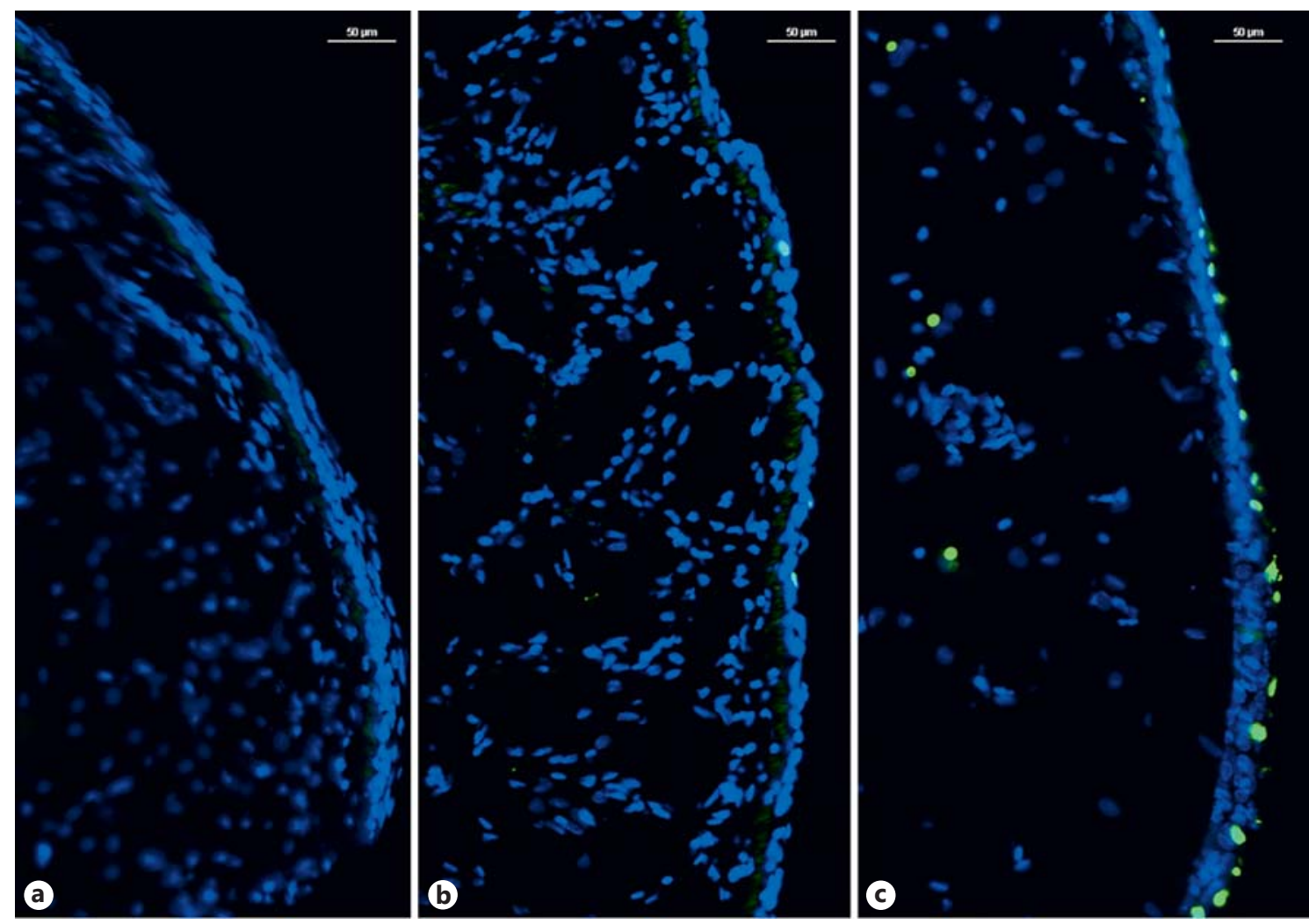

Fig. 9. Fluorescent-stained true vocal fold tissue specimens using TUNEL. Apical cell surface (on the right) of the true vocal fold epithelium after immobilization (a), approximation for $120 \mathrm{~min}$ (b) and vibration for $120 \mathrm{~min}$ (c). $\times 20$.

Table 3. Mean percentages of rated degree of TUNEL-positive staining in immobilized, approximated and vibrated vocal folds

\begin{tabular}{lcllll}
\hline Vocal fold condition & No TUNEL & $\begin{array}{l}\text { Minimal } \\
\text { TUNEL }\end{array}$ & $\begin{array}{l}\text { Moderate } \\
\text { TUNEL }\end{array}$ & $\begin{array}{l}\text { Abundant } \\
\text { TUNEL }\end{array}$ & Total \\
\hline Immobilized $(\mathrm{n}=2)$ & $50 \%$ & $0 \%$ & $50 \%$ & $0 \%$ & $100 \%$ \\
Approximated $(\mathrm{n}=5)$ & $40 \%$ & $40 \%$ & $20 \%$ & $0 \%$ & $100 \%$ \\
Vibrated $(\mathrm{n}=5)$ & $0 \%$ & $20 \%$ & $40 \%$ & $40 \%$ & $100 \%$ \\
\hline
\end{tabular}

cleavage of genomic DNA by DNase treatment. The positive control resulted in extensive nuclear fragmentation as indicated by the labeling of TUNEL in all vocal fold cell nuclei (fig. 10b). The majority of nuclear fragmentation was evident in epithelial cells, although scarce staining was also appreciated deeper in the primarily noncellular lamina propria.

\section{Discussion}

Epithelium, lamina propria and muscle comprise the true vocal fold [Hirano, 1974]. As the outermost portion of the vocal fold, the epithelium serves as a protective barrier between the lamina propria and the external environment. It is important to preserve epithelial barrier integrity to prevent and manage irregularities in this layered structure that could lead to a dysphonic voice such as 


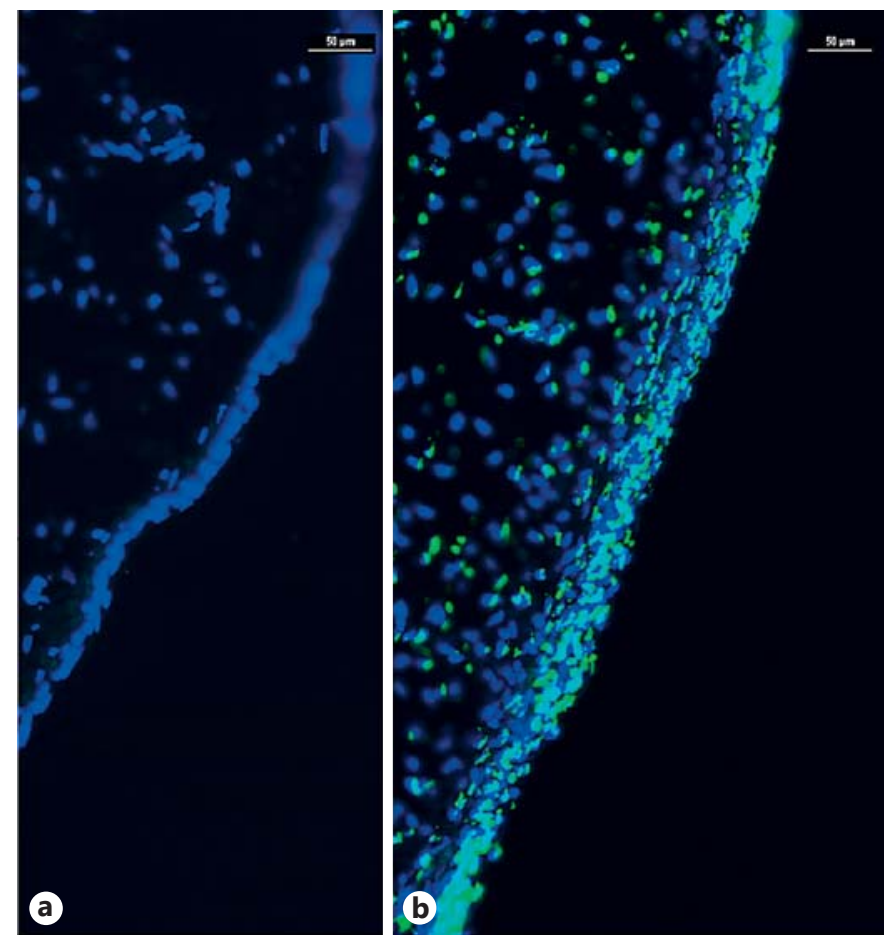

Fig. 10. Positive and negative controls of fluorescent-stained true vocal fold tissue specimens using the TUNEL method. A positive control of a normal vocal fold (a) and a negative control of a vocal fold specimen (b) exposed to $120 \mathrm{~min}$ of biomechanical vibration. The apical epithelial surfaces are on the right. $\times 20$.

hoarseness. During the life cycle of all cells, cell death is a regulated mechanism that eliminates unnecessary cells to maintain homeostasis. Previous studies revealed that vocal fold epithelial cells and fibroblasts undergo apoptotic cell death [Hellquist, 1997; Hirvikoski et al., 1999; Gaston et al., 2012; Bartlett et al., 2015; Lungova et al., 2015]. However, there is still limited acknowledgement of cell death as a consequence of different types of vocal fold damage and also a lack of consistent language to describe dying cells.

This study aimed to establish more systematic approaches to evaluate cell death in vocal fold epithelial cells and investigate cell death in response to immobilization, approximation and biomechanical vibration. Cell death was detected by morphological signs using TEM and in situ staining of DNA strand breaks with TUNEL. Ultrastructural evaluation revealed that apoptotic cell death is observed in the vocal fold epithelium of rabbits after undergoing approximation and vibration for $120 \mathrm{~min}$. Movement- and vibratory-induced apoptosis is primarily characterized by pyknotic epithelial nuclei, as indicated by condensed chromatin and apoptotic bodies. Although instances of necrosis are rare, morphological evidence of necrotic epithelial cells include cellular and organelle swelling (e.g. enlarged nuclei). Additionally, TUNEL confirmed that the vocal fold expresses an immunohistochemical marker for apoptosis along the apical surface of the epithelium.

We selected 2 complementary methods to measure vocal fold epithelial cell death. First, TEM was performed to provide high-resolution images of cellular structures. TEM was selected as the primary outcome because it serves as the gold standard technique for describing the ultrastructural features of cells committed to die [Elmore, 2007; Martinez et al., 2010]. In conjunction with TEM, TUNEL was performed on the laryngeal tissue sections. In cells undergoing apoptosis, caspase-activated DNase causes fragmented DNA that leads to a higher number of free $3^{\prime}$-hydroxyl ends $\left(3^{\prime}-\mathrm{OH}\right)$ in the nuclei. TUNEL is a common apoptosis assay that relies on labeling the $3^{\prime}-\mathrm{OH}$ with the tDt enzyme. While cells undergoing DNA fragmentation stain positive, normal or proliferating cells do not typically stain because they do not produce many 3 $\mathrm{OH}\left[\right.$ ApopTag ${ }^{\circledR}$ fluorescein in situ apoptosis detection kit, 2012]. Collectively, our findings suggest that vocal fold epithelial cells undergo cell death, primarily apoptosis, in response to vocal fold movement via adduction, abduction and biomechanical vibration. This is demonstrated by observations of chromatin condensation and apoptotic bodies according to TEM ratings and positively stained DNA fragmentation by TUNEL. When comparing the individual results of the TEM images and the fluorescent TUNEL-stained images, approximately half of the laryngeal tissue specimens showed similar patterns. That is to say there was a noted tendency for higher ratings of condensed chromatin and apoptotic bodies to correspond to a moderate-to-abundant number of TUNELpositive cells. Interestingly, the majority of specimens showing a consistent pattern between both analyses belonged to the vibration condition.

The 2 studied ultrastructural features of apoptotic cell death, condensed chromatin and apoptotic bodies, were rated as most prevalent following vocal fold approximation and vibration. These criteria indicate that multiple phases of apoptosis occurred. For instance, increased concentration of chromatin is an early stage of apoptosis in vocal fold epithelial cells, while a later stage is characterized by the formation of apoptotic bodies. A possible explanation for the occurrence of both features is that perhaps different locations of the epithelium may have 
undergone varying levels of biomechanical stresses that led to different stages of apoptosis. Interestingly, there were no identified inflammatory cells such as macrophages engulfing apoptotic bodies. This would be expected, given that the laryngeal tissues were harvested immediately after the experiments. Perhaps later stages of apoptosis would be observed with a longer delay between experimental procedures and tissue excision. In addition, necrotic cell death may be rarer after vocal fold movement and vibration, as few cells overall showed evidence of enlarged nuclei. However, episodes of some enlarged nuclei indicate that apoptosis may not be the only type of cell death in the vocal fold epithelium. It is possible that apoptosis and necrosis may occur simultaneously or that, given greater durations of trauma, apoptosis might eventually evolve into necrotic cell death.

In contrast, immobilized vocal folds were infrequently rated with features of cell death. Moreover, the morphology of immobilized vocal fold epithelium suggests that the primary cell shape was elongated or flattened, which indicates that the normal squamous epithelial cells remained intact. These findings in the immobilized experimental condition are not surprising, given that rabbits are relatively quiet animals and are not susceptible to the biomechanical stresses that occur during phonation. As such, the regular exchange of airflow through the glottis during respiration and vocal fold adduction during swallowing may not be significant factors in initiating cell death. We recognize that all epithelial cells in normal vocal folds must eventually die during turnover to promote a strong barrier. Moreover, it is likely that the rate of epithelial cell turnover occurs more gradually in normal vocal folds during immobilization. This preliminary notion might be interesting to consider the effect of vocal fold epithelial cell turnover on wound healing in patients who are prescribed voice rest.

There are acknowledged differences between the preliminary TEM qualitative analysis and the subsequent rating task of the TEM images. Although the qualitative portion described reduced epithelial cell nucleus size in response to vibration, the rating task disagreed with this assessment. In fact, the size of epithelial cells was rated consistently across all experimental conditions, and the immobilized vocal folds revealed a slightly greater occurrence of small nuclei. The discrepancy between the analyses could be explained by the inherent challenges with visual-perceptual judgements of TEM images. Based on these results, it is necessary to further explore alternative methods of measuring the size of the cell nuclei to evaluate its role in vocal fold cell death with improved accu- racy. Our findings suggest that a rounded cell shape may be common in vocal fold tissues exposed to vibration, so it may be that vibrated vocal folds demonstrate early morphological signs consistent with cell shrinkage by becoming more circular.

Ratings using the TUNEL method revealed that vocal folds exposed to approximation tended to demonstrate no-to-minimal TUNEL-positive staining, while vocal folds exposed to vibration demonstrated moderate-toabundant TUNEL-positive staining. These findings might be an initial indication that vibration leads to a greater expression of cell death via TUNEL. However, our rating procedure must be interpreted with caution, given that the fluorescent images were captured at different exposure times. To determine whether the studied conditions induced more or less labeling of TUNEL-positive cells, future research is needed to objectively quantify the degree of staining.

The effect of biomechanical vibration on cell death was previously examined in vocal fold fibroblasts, the primary cellular component of the lamina propria. Gaston et al. [2012], using a custom bioreactor in an immortalized cell line of human vocal fold fibroblasts after vibration exposure in a synthetic extracellular matrix scaffold, reported that apoptosis occurs in fibroblasts, although not at significantly high rates. This discrepancy could be based on methodological differences, as the vibratory stresses induced via a bioreactor may not be as representative of the high rate of vibratory cycles during in vivo phonation. However, we speculate that epithelial cells exposed to vibration using a bioreactor would possibly also result in apoptotic cell death. We acknowledge that the laryngeal cryosections in this study revealed damage to the lamina propria during tissue preparation, making it challenging to perform immunohistochemistry to assess cell death in this region. While exercising caution to preserve the epithelium while creating frozen sections, we neglected to consider preserving the remaining layers. Given the small size of rabbit larynges, this is an important limitation of performing frozen sections on vocal fold tissue. To address this concern, future immunohistochemical assays for cell death can be performed using thinner paraffinembedded tissue sections.

This study provides confidence that the movement of intermittent vocal fold adduction and abduction alone leads to some degree of cell death. Vibration also causes vocal fold epithelial cells to die. It remains unknown if cell death is considered a physiologic or pathologic condition or how irregular cell death influences the development of vocal fold disease. Our study simulated a condition of vi- 
bration that best represents the physiologic function of vocal fold vibration. This vibratory condition was selected based on previously reported morphological features of intact paracellular spaces and basement membrane, and a lack of functional tissue changes as measured by transepithelial resistance [Kojima et al., 2014a]. In contrast, raised intensity phonation was not studied due to evidence of pathologic damage, primarily epithelial tissue obligation, which could lead to less viable tissue for analysis [Kojima et al., 2014a]. We hypothesize that, during normal cycles of vibration, biomechanically induced stresses signal vocal fold epithelial cells to undergo apoptosis in order to regularly renew the tissue and maintain homeostasis, as has been reported in the intestinal epithelium [Marchiando et al., 2011]. We believe that cell death is a necessary process that assists with maintaining a functional vocal fold epithelial barrier. However, excessive or deficient rates of apoptosis have been observed in a variety of diseases including cancer and autoimmune and neurological diseases [Ashkenazi and Dixit, 1998; Cory and Adams, 2002]. The study of cell death has substantial potential to inform current research about vocal fold wound healing and epithelial diseases such as laryngeal squamous cell carcinoma or benign nodular formations.

While vocal fold epithelial cells die, we do not yet understand the functional consequences. Transepithelial resistance, a valuable functional measure of barrier integrity, reflects intracellular as well as intercellular changes. Because changes in resistance often correspond to the tight intercellular junction, cell death alone may less likely contribute to differences in resistance values. During apoptosis in the small intestine, the tight and adherens junction proteins relocate from the apical toward the lateral cell membrane [Marchiando et al., 2011]. This active, tight intercellular rearrangement might serve to maintain the epithelial barrier [Madara, 1990; Marchiando et al., 2011] and would likely result in no differences in transepithelial resistance. Additional research is needed to assess the cellular response to replace eliminated cells, such as proliferation and differentiation, to enhance our understanding about vocal fold cell turnover rates. Given that the laryngeal specimens were excised immediately after vibration, we speculate that cell proliferation would require additional time during recovery following movement- and vibratory-induced injury.

Although previous studies alluding to vocal fold cellular degradation after injury or in vocal fold disease are highly suggestive of cell death [Gray and Titze, 1988; Kotby et al., 1988; Dikkers et al., 1993; Marcelino and Oliveira, 2005; Martins et al., 2010, 2011; Rousseau et al., 2011], it is not possible to confirm this without more concrete methods. Our results provide an important step toward better communicating the biochemical mechanism of cell death in the vocal fold. It is recognized that future research is necessary to quantify the rate of apoptosis with greater durations of damage exposure to determine if cell death increases or remains a static response. However, our findings lay a preliminary foundation for future studies to quantify cell death as it may relate to different degrees of vocal fold damage. The methods that we used to measure apoptotic events may also become useful biomarkers for the diagnosis and prognosis of human vocal fold disease.

\section{Acknowledgements}

This research was supported by the National Institute on Deafness and Other Communication Disorders of the National Institutes of Health (NIH) under award No. F31DC014621 and No. R01DC011338. The content is solely the responsibility of the authors and does not necessarily represent the official views of the $\mathrm{NIH}$. Experiments and data analysis of transmission electron microscopy images were performed in part through the use of the Vanderbilt University Medical Center Cell Imaging Shared Resource, which is supported by the NIH under award Nos. CA68485, DK20593, DK58404, DK59637 and EY08126.

\section{Disclosure Statement}

None of the authors have any financial interests from any companies or other entities that have an interest in the information in this work to report. There are no conflicts of interest to disclose.

ApopTag ${ }^{\circledR}$ fluorescein in situ apoptosis detection
kit (2012).
Ashkenazi, A., V.M. Dixit (1998) Death receptors:
signaling and modulation. Science 281: 1305-
1308.
Bartlett, R.S., J.D. Gaston, T.Y. Yen, S. Ye, C. Ken-
dziorski, S.L. Thibeault (2015) Biomechanical
screening of cell therapies for vocal fold scar.
Tissue Eng Part A 21: 2437-2447.
Cory, S., J.M. Adams (2002) The Bcl2 family: reg-
ulators of the cellular life-or-death switch.
Nat Rev Cancer 2: 647-656.
Denecker, G., D. Vercammen, W. Declercq, P.
Vandenabeele (2001) Apoptotic and necrotic
cell death induced by death domain receptors.
Cell Mol Life Sci 58: 356-370.
Dikkers, F.G., C.E. Hulstaert, J.A. Oosterbaan, F.J.
Cervera-Paz (1993) Ultrastructural changes
of the basement membrane zone in benign le-
sions of the vocal folds. Acta Otolaryngol 113:
98-101.


Elmore, S. (2007) Apoptosis: a review of programmed cell death. Toxicol Pathol 35: 495516.

Gaston, J., B. Quinchia Rios, R. Bartlett, C. Berchtold, S.L. Thibeault (2012) The response of vocal fold fibroblasts and mesenchymal stromal cells to vibration. PloS One 7: e30965.

Ge, P.J., L.C. French, T. Ohno, D.L. Zealear, B. Rousseau (2009) Model of evoked rabbit phonation. Ann Otol Rhinol Laryngol 118: 51-55.

Gray, S.D. (2000) Cellular physiology of the vocal folds. Otolaryngol Clin North Am 33: 679698.

Gray, S., I. Titze (1988) Histologic investigation of hyperphonated canine vocal cords. Ann Otol Rhinol Laryngol 97: 381-388.

-Hellquist, H.B. (1997) Apoptosis in epithelial hyperplastic laryngeal lesions. Acta Otolaryngol Suppl 527: 25-29.

Hirano, M. (1974) Morphological structure of the vocal cord as a vibrator and its variations. Folia Phoniatr 26: 89-94.

Hirvikoski, P., E. Kumpulainen, J. Virtaniemi, R. Pirinen, L. Salmi, P. Halonen, R. Johansson, V.M. Kosma (1999) Enhanced apoptosis correlates with poor survival in patients with laryngeal cancer but not with cell proliferation, Bcl-2 or p53 expression. Eur J Cancer 35: 231237.

Hooper, C.E. (1956) Cell turnover in epithelial populations. J Histochem Cytochem 4: 531540
Kojima, T., C.V. Valenzuela, C.K. Novaleski, M Van Deusen, J.R. Mitchell, C.G. Garrett, M.P. Sivasankar, B. Rousseau (2014a) Effects of phonation time and magnitude dose on vocal fold epithelial genes, barrier integrity, and function. Laryngoscope 124: 2770-2778.

Kojima, T., M. Van Deusen, W.G. Jerome, C.G. Garrett, M.P. Sivasankar, C.K. Novaleski, B. Rousseau (2014b) Quantification of acute vocal fold epithelial surface damage with increasing time and magnitude doses of vibration exposure. PLoS One 9: e91615.

Kotby, M.N., A.M. Nassar, E.I. Seif, E.H. Helal, M.M. Saleh (1988) Ultrastructural features of vocal fold nodules and polyps. Acta Otolaryngol 105: 477-482.

Lalaoui, N., L.M. Lindqvist, J.J. Sandow, P.G. Ekert (2015) The molecular relationships between apoptosis, autophagy and necroptosis. Semin Cell Dev Biol 39: 63-69.

Lungova, V., J.M. Verheyden, J. Herriges, X. Sun, S.L. Thibeault (2015) Ontogeny of the mouse vocal fold epithelium. Dev Biol 399: 263-282.

Madara, J.L. (1990) Maintenance of the macromolecular barrier at cell extrusion sites in intestinal epithelium: physiological rearrangement of tight junctions. J Membr Biol 116: 177-184.

Majno, G., I. Joris (1995) Apoptosis, oncosis, and necrosis. An overview of cell death. Am J Pathol 146: 3-15.

Marcelino, F.C., D.T. Oliveira (2005) Histopathological changes of vocal folds induced by chronic pollutant exposure: an experimental study. J Voice 19: 529-533.
Marchiando, A.M., L. Shen, W.V. Graham, K.L. Edelblum, C.A. Duckworth, Y. Guan, M.H. Montrose, J.R. Turner, A.J. Watson (2011) The epithelial barrier is maintained by in vivo tight junction expansion during pathologic intestinal epithelial shedding. Gastroenterology 140: 1208-1218.e1201-1202.

Martinez, M.M., R.D. Reif, D. Pappas (2010) Detection of apoptosis: a review of conventional and novel techniques. Anal Methods 2: 9961004.

Martins, R.H., J. Defaveri, M.A. Custodio Domingues, E.S.R. de Albuquerque, A. Fabro (2010) Vocal fold nodules: morphological and immunohistochemical investigations. J Voice 24: 531-539.

Martins, R.H., J. Defaveri, M.A. Domingues, R. de Albuquerque e Silva (2011) Vocal polyps: clinical, morphological, and immunohistochemical aspects. J Voice 25: 98-106.

-Pellettieri, J., A. Sanchez Alvarado (2007) Cell turnover and adult tissue homeostasis: from humans to planarians. Ann Rev Genet 41: 83-105.

Rousseau, B., A. Suehiro, N. Echemendia, M. Sivasankar (2011) Raised intensity phonation compromises vocal fold epithelial barrier integrity. Laryngoscope 121: 346-351.

-Van Den Berg, J. (1958) Myoelastic-aerodynamic theory of voice production. J Speech Hearing Res 1: 227-244.

Watson, A.J., C.A. Duckworth, Y. Guan, M.H. Montrose (2009) Mechanisms of epithelial cell shedding in the mammalian intestine and maintenance of barrier function. Ann NY Acad Sci 1165: 135-142. 